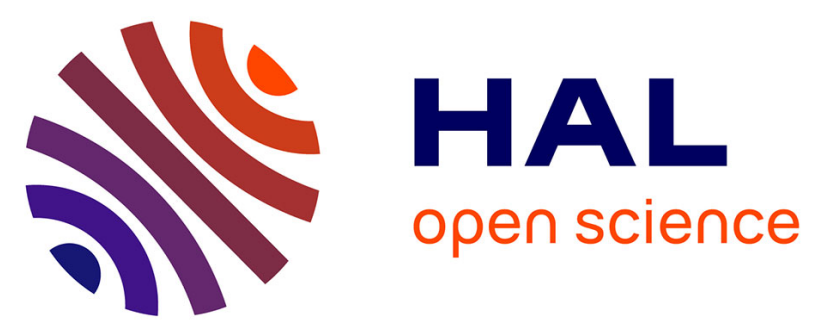

\title{
A Combination of Single Nucleotide Polymorphisms is Associated with the Interindividual Variability of Cholesterol Bioavailability in Healthy Adult Males
}

Charles Desmarchelier, Estelle Wolff, Catherine Defoort, Marion Nowicki, Pierre-emmanuel Morange, Marie-Christine Alessi, René Valéro, Alain

Nicolay, Denis Lairon, Patrick Borel

\section{To cite this version:}

Charles Desmarchelier, Estelle Wolff, Catherine Defoort, Marion Nowicki, Pierre-emmanuel Morange, et al.. A Combination of Single Nucleotide Polymorphisms is Associated with the Interindividual Variability of Cholesterol Bioavailability in Healthy Adult Males. Molecular Nutrition and Food Research, 2020, 64 (22), pp.2000480. 10.1002/mnfr.202000480 . hal-03155917

\author{
HAL Id: hal-03155917 \\ https://hal.inrae.fr/hal-03155917
}

Submitted on 9 Mar 2021

HAL is a multi-disciplinary open access archive for the deposit and dissemination of scientific research documents, whether they are published or not. The documents may come from teaching and research institutions in France or abroad, or from public or private research centers.
L'archive ouverte pluridisciplinaire HAL, est destinée au dépôt et à la diffusion de documents scientifiques de niveau recherche, publiés ou non, émanant des établissements d'enseignement et de recherche français ou étrangers, des laboratoires publics ou privés. 
A combination of single nucleotide polymorphisms is associated with the interindividual variability of cholesterol bioavailability in healthy adult males.

Charles Desmarchelier, ${ }^{1 *}$ Estelle Wolff, ${ }^{1}$ Catherine Defoort, ${ }^{1}$ Marion Nowicki, ${ }^{1}$ PierreEmmanuel Morange, ${ }^{1}$ Marie-Christine Alessi, ${ }^{1}$ René Valéro, ${ }^{1,2}$ Alain Nicolay, ${ }^{1}$ Denis Lairon, ${ }^{1}$ and Patrick Borel ${ }^{1 *}$

${ }^{1}$ C2VN, INRAE, INSERM, Aix Marseille Univ, Marseille, France

${ }^{2}$ APHM, CHU Conception, Department of Nutrition, Metabolic Diseases and Endocrinology, Marseille, France

* To whom correspondence should be addressed:

Charles.Desmarchelier@univ-amu.fr; Patrick.Borel@univ-amu.fr

UMR 1263 INSERM/1260 INRA/Université d'Aix-Marseille

Centre CardioVasculaire et Nutrition (C2VN)

Faculté de Médecine

27 boulevard Jean Moulin

13385 Marseille cedex 5

France

Phone: +33(0)4 91324282

Abbreviations: CVD, cardiovascular diseases; D7, heptadeuterated; NPC1L1, NPC1 like intracellular cholesterol transporter 1; PLS, partial least squares; SNP, single nucleotide polymorphism; TC, total cholesterol; VIP, variable importance in the projection. A complete list of gene names and symbols can be found in Supplemental Table 1.

Keywords: cardiovascular diseases; blood lipids; genetic variation; absorption; nutrigenetics. 


\begin{abstract}
Scope: Cholesterol bioavailability displays a high interindividual variability, partly due to genetic factors. Existing studies have focused on single nucleotide polymorphisms (SNPs) analysed individually, which only explained a minor fraction of the variability of this complex phenotype. We aimed to identify a combination of SNPs associated with a significant part of the variability in cholesterol bioavailability.
\end{abstract}

Methods and results: Thirty-nine healthy adult males were given a standard test snack containing $80 \mathrm{mg}$ heptadeuterated (D7) cholesterol. The plasma D7-cholesterol concentration was measured at equilibrium $40 \mathrm{~h}$ after test snack intake. The D7-cholesterol response (D7cholesterol/total cholesterol concentration) exhibited a relatively high interindividual variability $(\mathrm{CV}=32 \%)$. The association of exonic SNPs in candidate genes (188 genes involved in or related to cholesterol metabolism) with the plasma D7-cholesterol response was assessed by univariate statistics followed by partial least squares regression. Data obtained allowed us to generate a significant model ( $\mathrm{p}$-value after cross-validation ANOVA $=1.64 \times 10^{-7}$ ) that included 8 SNPs (SOAT2-rs9658625, DNAH11-rs11768670, LIPC-rs690, MVK-rs2287218, GPAM-rs10787428, $A P O E$-rs7412, $C B S$-rs234706 and $W R N$-rs1801196) which explained 59.7\% of the variance in cholesterol bioavailability (adjusted $\mathrm{R}^{2}$ ).

Conclusion: We here report a combination of SNPs associated with a significant part of the variability in dietary cholesterol bioavailability.

This trial was registered at http://www.clinicaltrials.gov as NCT02100774. 


\section{INTRODUCTION}

Prolonged exposure to high blood cholesterol concentrations has been associated with an increased risk to develop cardiovascular diseases (CVD) later in life [1, 2], underlining the need for early detection of hypercholesterolemia and reasoned choice of relevant dietary and pharmacological treatment strategies. Patients with high intestinal cholesterol absorption, who might represent as much as $25 \%$ of the population, respond poorly to statin treatment, which inhibits the rate-limiting enzyme of cholesterol synthesis, and have a higher risk of recurrence of major coronary events [3]. In order to improve their outcome and minimize treatment cost [4], these patients should receive tailored dietary recommendations, e.g. plant sterols and stanols $[5,6]$ and/or lower dietary cholesterol intake [7], and if insufficient, pharmacological treatments [8], e.g. ezetimibe [9] or bile acid sequestrants [10]. Targeting of intestinal cholesterol absorption has recently been shown to be a valuable approach in CVD risk reduction. Indeed, the addition of ezetimibe, which inhibits NPC1 like intracellular cholesterol transporter 1 (NPC1L1), the main protein involved in the apical transport of cholesterol in the small intestine [11], to a statin therapy resulted in a further reduction of LDL-cholesterol (LDL-C) concentrations and cardiovascular event risk in patients with a recent acute coronary syndrome [12]. Recently, ezetimibe monotherapy was shown to lead to a decrease in the incidence of a composite of sudden cardiac death, myocardial infarction, coronary revascularization, or stroke in patients aged $\geq 75$ years with elevated LDL-C concentration, together with a decrease in LDLC concentration [13].

Although a healthy diet is widely acknowledged as a cornerstone of disease prevention and management [14], its optimal composition, in terms of specific foods or isolated nutrients, such as cholesterol, is still a matter of great debate. This is notably due to the fact that intestinal cholesterol absorption efficiency displays a high interindividual variability, with values ranging between 30 and $80 \%$ [15], assumed to be due to both dietary factors (e.g. dietary fat and cholesterol, fibres, plant sterols) and host-related factors [16], including the gut microbiota [17] and genetic factors $[18,19]$. Although some SNPs involved in this process have been identified, the explained variability remains low since existing studies have focused on few candidate SNPs [20] or rare mutations [21]. Yet, this process is a complex phenotype, which is likely to involve numerous genes. We thus assumed that a significant part of its variability is explained by the additive effect of several genotypes which, taken individually, may have a small, barely significant effect. Our aim was thus to identify a combination of SNPs significantly associated with the interindividual variability in cholesterol bioavailability using D7-cholesterol as an acknowledged marker of cholesterol absorption. 


\section{SUBJECTS AND METHODS}

\subsection{Reagents}

D7-cholesterol $\left(25,26,26,26,27,27,27-\mathrm{H}^{7} ; 99 \%\right.$ enrichment) was supplied by CDN (Pointe Claire, QC, Canada); epicoprostanol and methylsilyltrifluoroacetamide were from Sigma (Saint-Quentin-Fallavier, France); absolute ethanol, hexane and pyridine were from Carlo Erba Reagents S.A.S. (Peypin, France). All chemicals used in this study were of analytical grade.

\subsection{Participants}

Forty healthy, non-obese (BMI $<30 \mathrm{~kg} / \mathrm{m}^{2}$ ), non-smoking male adults were recruited for the study. Participants reported normal energy intake with $<2 \%$ alcohol as total energy intake. Participants had no history of chronic disease, hyperlipidaemia, or hyperglycaemia and were not taking any medication known to affect lipid metabolism the month before the study or during the study period. Because of the relatively large volume of blood collected during the study, blood haemoglobin concentration $>13 \mathrm{~g} / \mathrm{dL}$ was an inclusion criteria. The study was approved by the regional committee on human experimentation $\left(\mathrm{N}^{\circ} 2008-\mathrm{A} 01354-51\right.$, Comité de Protection des Personnes Sud Méditerranée I, France). Procedures followed were in accordance with the Declaration of Helsinki of 1975 as revised in 1983. Objectives and requirements of the study were fully explained to all participants before beginning the study, and written informed consent was obtained from each subject. One subject left the study for personal reasons before he participated in the cholesterol-feeding experiment, which left 39 participants whose baseline characteristics are reported in Table 1.

\subsection{Cholesterol-feeding trial}

In order to compare cholesterol bioavailability between participants, plasma D7cholesterol concentrations were measured $40 \mathrm{~h}$ after consumption of a standardized test snack containing D7-cholesterol [22]. After an $8 \mathrm{~h}$ fast, a baseline blood sample was taken from the participants via glass tubes containing EDTA-K 3 at the local Center for Clinical Investigation (Hôpital de la Conception, Marseille, France). Plasma was separated from whole blood by centrifugation $\left(2000 \mathrm{~g}\right.$ for $10 \mathrm{~min}$ at $20^{\circ} \mathrm{C}$ ) and frozen at $-20^{\circ} \mathrm{C}$ until further analysis. Eighty $\mathrm{mg}$ D7-cholesterol was incorporated as a powder in $25 \mathrm{~g}$ butter melted at $45^{\circ} \mathrm{C}$ in a microwave oven and the mixture was homogenized until solidification at room temperature. Participants then consumed a standardized test snack that comprised $25 \mathrm{~g}$ butter spread on $80 \mathrm{~g}$ of bread slices, 
to which $30 \mathrm{~g}$ jam could be added, and were allowed to drink only water. Two days later, they were invited to return in the fasting state at the Center for Clinical Investigation where a blood sample was collected $40 \mathrm{~h}$ following the ingestion of D7-cholesterol. Indeed, it has been previously shown that the area under the curve of the plasma free cholesterol enrichment of a single isotope labelled cholesterol tracer was well correlated with the cholesterol absorption rate as measured by the plasma dual stable-isotope ratio method over a $24-96 \mathrm{~h}$ period following its intake [23]. Moreover, we have shown that the plasma D7-cholesterol concentration plateaued $24 \mathrm{~h}$ after consumption of a standardized test snack containing D7-cholesterol and that it remained at this equilibrium level up to $48 \mathrm{~h}$ after consumption of the standardized snack [22].

\subsection{Chemical analyses}

Fasting plasma total cholesterol (TC), HDL-cholesterol, LDL-C, triglyceride, glucose and haemoglobin concentrations were measured before consumption of the test snack with standard methods: analyte concentrations were measured on a Modular ${ }^{\circledR}$ PP instrument (Roche Diagnostics, Meylan, France), except for haemoglobin which was measured on an ADVIA $120 \circledR$ instrument (Siemens Healthcare Diagnostics, Saint-Denis, France) at La Conception Hospital (Biochemistry Laboratory, Marseille). All analyses were performed according to the manufacturer's instructions.

D7-cholesterol concentration was measured as previously described [24]. Briefly, $200 \mu \mathrm{L}$ epicoprostanol ( $5 \mu \mathrm{mol} / \mathrm{L}$ ), used as internal standard, was added to $200 \mu \mathrm{L}$ plasma. After alkaline hydrolysis using 6.4 M KOH and extraction (water/hexane; $2 / 5 \mathrm{~mL}$ ), samples were derivatised with a methylsilyltrifluoroacetamide/pyridine mixture $(40 / 50 \mu \mathrm{L})$ for $60 \mathrm{~min}$ at $70^{\circ} \mathrm{C}$. After evaporation, the dry residue was dissolved in $500 \mu \mathrm{L}$ hexane and a volume of 2 $\mu \mathrm{L}$ was injected in a gas chromatography-mass spectrometer (Hewlett-Packard 6890 GC-5973 MS). Samples were analysed on a quadrupole mass spectrometer in Single Ion Monitoring mode (ions m/z 336 and m/z 370 were chosen for D7-cholesterol and epicoprostanol quantification respectively). Plasma D7-cholesterol enrichment was calculated by comparison to a standard curve with internal standard and was expressed as $\mu \mathrm{mol} \mathrm{D} 7$-cholesterol/mmol cholesterol, as usual [25].

\subsection{DNA preparation and genotyping methods}

An average of $25 \mu \mathrm{g}$ of DNA was isolated from saliva samples using the Oragene kit (DNA Genotek, Ottawa, Ontario, Canada). DNA preparation and genotyping methods were performed as previously described [26]. The whole genome was genotyped using 
HumanOmniExpress BeadChips (Illumina), which allow for the analysis of $\approx 7.33 \times 10^{5}$ SNPs/DNA sample.

\subsection{Choice of candidate genes and SNPs}

Literature search in PubMed for genes whose SNPs have been shown to be associated with cholesterolemia variability and analysis of pathways involved in cholesterol absorption or metabolism resulted in the selection of 188 genes (Supporting Information Table S1). SNP selection is summarized in Supporting Information Figure S1. Due to the relatively small size of our study population, we narrowed our analysis to exonic SNPs (360 SNPs expressed on the arrays, out of 11747 in total; National Center for Biotechnology Information annotation as provided by Illumina). SNPs for which the genotype call rate was $<95 \%$ and SNPs that presented a significant departure from the Hardy-Weinberg equilibrium $(p<0.05$; Chi-squared test) were excluded from all subsequent analyses (20 SNPs excluded), leaving 340 SNPs. SNPs were tested under both additive and dominant models [27-29]. The additive model assumes that there is a uniform, linear increase or decrease in the quantitative trait for each copy of the minor allele. For SNPs under the dominant model, participants homozygous for the lesser frequent allele were grouped with heterozygous participants and were compared with participants homozygous for the more frequent allele. SNPs with fewer than 5 observations in the minor group were excluded from further analysis, leaving 46 and 172 SNPs in the additive and dominant model, respectively. Moreover, for each candidate gene, when two SNPs were in perfect linkage disequilibrium $\left(\mathrm{R}^{2}=1\right)$, one was randomly excluded from further analysis, leaving 44 and 156 SNPs (in 76 genes) for statistical analysis in the additive and dominant model, respectively. Linkage disequilibrium information was retrieved using the LD TAG SNP Selection tool from the SNPinfo Web Server (https://snpinfo.niehs.nih.gov/snpinfo/snptag.html) considering genotype data from dbSNP in the European population.

\subsection{Calculations and statistical analysis}

Data were expressed as mean \pm SEM. Normality was assessed using the KolmogorovSmirnov and the Shapiro-Wilk tests. Statistical dependence between two variables was assessed by Pearson's correlation coefficient. Differences in fasting TC concentration before and 40h after intake of the D7-cholesterol test snack were tested by paired t-test. For all tests, the bilateral alpha risk was $\alpha=0.05$. Statistical analyses were performed using IBM SPSS Statistics (Version 20.0; Armonk, NY, U.S.A.). 


\section{Statistics: PLS regression}

To identify SNPs associated with the variability observed in cholesterol bioavailability, we calculated the D7-cholesterol/TC ratio (see Results for an explanation), hereafter named the D7-cholesterol response. In a first approach, we performed univariate analyses to compare the D7-cholesterol response between subgroups of participants who bore different genotypes at the candidate SNPs, using Plink (v1.07, http://pngu.mgh.harvard.edu/purcell/plink/) [30]. SNPs that exhibited a p-value $<0.05$ (Wald test asymptotic p-value) were selected for partial least squares (PLS) regression analysis, which is the multivariate regression extension of principal component analysis [31], following previously published rationale and model assumptions [32, 33]. First, a PLS regression model including all selected SNPs coded in units of variance was built. Variables were ranked according to their variable importance in the projection (VIP) value and several PLS regression models were then built using increasing VIP threshold values. The model maximizing the adjusted explained variance (adjusted $\mathrm{R}^{2}$ ) was selected (32). The adjusted $\mathrm{R}^{2}$ was calculated according to Equation 1:

$$
\text { Adjusted } R^{2}=1-\frac{\left(1-R^{2}\right)(n-1)}{n-k-1} \quad \text { (Equation 1) }
$$

With $n$ the sample size and $k$ the number of predictors in the model (excluding the constant). SIMCA-P13 software (Umetrics, Umeå, Sweden) was used for all multivariate data analyses and modelling. Additional validation criteria and procedures of the PLS regression models are described in Supporting Information Methods. 


\section{RESULTS}

\subsection{Plasma D7-cholesterol response of the participants}

Fasting plasma TC concentration of the participants did not change over the course of the experiment $(4.33 \pm 0.16$ vs $4.31 \pm 0.14 \mathrm{mmol} / \mathrm{L}$ at $\mathrm{t} 0$ and $\mathrm{t}+40 \mathrm{~h}$ respectively; $p=0.949$, paired t-test). The plasma D7-cholesterol concentration of the participants at $\mathrm{t}+40 \mathrm{~h}$ did not significantly correlate with any of the reported characteristics of the participants, except with TC (Pearson's $\mathrm{r}=0.513 ; p=0.001$ ) and LDL-C concentration (Pearson's $\mathrm{r}=0.638 ; p<0.001$ ). Thus, in order to normalize the D7-cholesterol concentration to the cholesterol pool of each subject, we calculated the D7-cholesterol response as the D7-cholesterol/TC ratio ( $\mu \mathrm{mol} / \mathrm{mmol})$ [25] (Figure 1). It followed a normal distribution ( $p=0.20$ and $p=0.91$ following the Kolmogorov-Smirnov and the Shapiro-Wilk test respectively; skewness=0.221 and kurtosis=0.209). The CV of the D7-cholesterol response was $32 \%$ and the ratio between the highest and the lowest responder was $\approx 4.9$.

\subsection{Genetic variants associated with the D7-cholesterol response}

We measured the association between exonic SNPs and the interindividual variability in the D7-cholesterol response. Five and nine SNPs exhibited a significant p-value under the additive and dominant model, respectively (Table 2). Under the additive model, WRNrs1801195 and $W R N$-rs 1800392 were in strong linkage disequilibrium $\left(\mathrm{R}^{2}=0.945\right)$. Under the dominant model, the three SNPs in DNAH11 were not in linkage disequilibrium (maximum $\mathrm{R}^{2}=0.307$ ). Unstandardized regression coefficients ( $B$ coefficients), which represent the mean change in the D7-cholesterol response for each additional copy of the minor allele under the additive model and in the presence of the minor allele under the dominant model are also provided in Table 2.

\subsection{Combination of genetic variants associated with the D7-cholesterol response}

We first entered all fourteen significant SNPs into PLS regression analysis. From the first model thus obtained, SNPs were ranked by decreasing variable importance in the projection (VIP) value, which estimates the contribution of each SNP in the projection used in the PLS regression model, and thus in the studied phenotype. When two SNPs were in linkage disequilibrium (i.e. WRN-rs1801195 and WRN-rs1800392, $\mathrm{R}^{2}=0.95$ ), the SNP exhibiting the lowest VIP value was excluded. When the same SNP was entered coded under both additive and dominant models (i.e. LIPC-rs690), the SNP under the genetic model with the lowest VIP value was excluded. The model obtained explained $63.8 \%$ of the variance $\left(\mathrm{R}^{2}\right)$, i.e. of the 
interindividual variability of the D7-cholesterol response, with an adjusted $\mathrm{R}^{2}$ of 0.491 and the $\mathrm{R}^{2}$ after cross-validation was 0.521 (Supporting Information Table S2 and Supporting Information Methods). To improve the model and find a combination of SNPs explaining a higher percentage of the variance in the D7-cholesterol response, we generated new models by sequentially excluding the SNP that displayed the lowest VIP value and we finally selected the model maximising the adjusted $\mathrm{R}^{2}$ and the $\mathrm{R}^{2}$ after cross validation (Supporting Information Table S2). The retained model included 8 SNPs, SOAT2-rs9658625, DNAH11-rs11768670, $L I P C$-rs690, $M V K$-rs2287218, GPAM-rs10787428, APOE-rs7412, CBS-rs234706, and WRNrs1801196 (Table 3 and Figure 2). It explained 68.2\% of the variance $\left(\mathrm{R}^{2}\right)$ and had an adjusted $\mathrm{R}^{2}$ of 0.597 and a $\mathrm{R}^{2}$ after cross-validation of 0.580 (p-value after cross-validation ANOVA $=1.64 \times 10^{-7}$ ) (Table 3). The robustness and the stability of the model was validated by three additional methods (Supporting Information Methods, Table S3, Figure S2 and Figure S3). With the knowledge of a participant genotype at the 8 SNPs in the selected model, it was possible to calculate the participant D7-cholesterol response using the following equation:

$$
\mathrm{D} 7=1.222+\sum_{1}^{8} \mathrm{ri} \times \text { number of minor allele (Equation 2) }
$$

with D7 the plasma D7-cholesterol response, $r_{i}$ the unstandardized regression coefficient of the $i^{\text {th }}$ SNP in the PLS regression model (provided in Table 3). When SNPs were entered under the dominant model, participants homozygous for the lesser frequent allele were grouped with heterozygous participants and the number of minor alleles for both these groups was considered to be 1 . 


\section{DISCUSSION}

In this study in 39 healthy adult males, using a multivariate statistical approach, we showed that the relatively high interindividual variability in intestinal cholesterol absorption efficiency $(\mathrm{CV}=32 \%)$ was significantly associated with a combination of 8 exonic SNPs in genes involved in lipid transport and metabolism $\left(\mathrm{R}^{2}=0.597\right)$. In order to estimate cholesterol bioavailability we measured the plasma concentration of a stable isotope-enriched cholesterol moiety, D7-cholesterol, ingested together with a standardized test snack. To reach the general circulation, cholesterol is first packed by the small intestine into chylomicrons, which are secreted into the lymph during several sequential meals [22]. To account for this kinetics, plasma D7-cholesterol concentration was measured $40 \mathrm{~h}$ after its intake, i.e. at its steady state in the plasma, as previously shown [15, 23, 24, 34]. Several methods exist to assess intestinal cholesterol absorption, as reviewed elsewhere [35]. The use of a single stable isotope [23, 36] offers the advantage of being direct (which is not the case when using plant sterol as a marker), safe (especially against radioisotopic methods), relatively non-invasive (a single blood collection is required), it does not require stool collection and its steady-state plasma concentration correlates well with intestinal cholesterol absorption when compared with sophisticated radioisotopic methods [23]. However, its relatively high price limits its applicability to small studies.

The first noteworthy observation was the partial positive correlation between the D7cholesterol concentration of the participants and their fasting LDL-C concentration, an independent risk factor for CVD [37]. This is in agreement with results from a random sample in the Finnish population, where cholesterol fractional absorption was measured by the peroral double-isotope continuous cholesterol-betasitosterol feeding method [38], and with results from the Ludwigshafen Risk and Cardiovascular health study, where cholesterol absorption was estimated by measuring plasma plant sterol concentrations [39]. Nonetheless, the effect of a high cholesterol absorption phenotype on CVD is not solely mediated by its effect on LDL-C concentration since other studies have shown a significant association between high cholesterol absorption and CVD while LDL-C concentrations remained unchanged [3, 40]. This highlights the relevance of the identification of the absorber/synthesizer phenotype in primary or secondary prevention of CVD, beyond simple CVD risk estimation based on LDL-C concentration [8]. 
The complexity of the relationship between intestinal cholesterol absorption and LDL-C concentration might be due to the complexity of cholesterol metabolism. Indeed, circulating cholesterol originates both from de novo synthesis and from dietary intakes, which vary widely between individuals according to their own de novo synthesis and their dietary habits. In addition, cholesterol de novo synthesis and cholesterol absorption exhibit a reciprocal relationship [38, 41]. It should also be stressed that cholesterol absorbed at the intestinal level has two origins, namely dietary cholesterol and cholesterol from bile, which is actually the main contributor to the intraluminal cholesterol pool [18]. Finally, cholesterol absorption displays a high interindividual variability [15]. Our study confirms this key trait since the CV of the D7cholesterol response was $32 \%$ herein, in line with that observed by Bosner et al. in thirty-two healthy males (CV of $21 \%$ as calculated by the present authors) [15]. Although it is well established that an increase in dietary cholesterol leads on average to a relatively modest increase in LDL-C [42, 43], whether it translates into increased CVD risk remains a matter of great debate. A meta-analysis of forty studies and nineteen trials did not reveal any significant association with CVD risk [42]. Recently, two large studies reported opposing results concerning the association between egg intake and LDL-C concentrations or CVD risk $[44,45]$. Although the discrepancy in results can be due to errors inherent to food frequency questionnaires in epidemiological studies, another possible explanation is unadjusted residual confounding. It is fairly conceivable that there could be a significant effect of the interaction of the cholesterol absorber/synthesizer phenotype with the impact of dietary cholesterol intakes on LDL-C concentration and thus CVD risk. If verified, this hypothesis could lead to personalized dietary recommendations, based on this phenotype, targeting either cholesterol absorption or synthesis in order to decrease CVD risk.

We showed that the interindividual variability in intestinal cholesterol absorption efficiency is notably due to genetic variations and that a combination of 8 exonic SNPs is significantly associated with this variability. Following candidate SNPs approaches, a few genetic variations have been previously reported [46], namely SNPs in $A B C G 5$ and 8 [20, 47], $A B O$ [20], NPC1L1 [48] or APOE [49]. Since cholesterol bioavailability is a complex process that involves numerous genes [18], a thorough study of the association between candidate genetic variants and this phenotype should ideally include SNPs in all genes that are assumed to be involved in this phenotype. Following thorough pathway analysis and literature search, we selected 188 candidate genes (Supporting Information Table S1), which allowed us to investigate the effect of 156 exonic SNPs on cholesterol absorption efficiency. The results of 
our statistical analysis first allowed the identification of 14 SNPs associated individually with the variability of the D7-cholesterol response between the study participants. We then applied a multivariate regression analysis, PLS regression, to determine which combination of these SNPs could maximize the explained variance of the studied phenotype. Our analysis, whose validity was checked by several tests, showed that a combination of 8 SNPs was significantly associated with the interindividual variability in the D7-cholesterol response (adjusted $\mathrm{R}^{2}=$ 0.597). For $L I P C$-rs690, GPAM-rs10787428, APOE-rs7412 and $W R N$-rs1801196, the presence of the minor allele was associated with an increase in intestinal cholesterol absorption efficiency, as illustrated by a positive regression coefficient, while for SOAT2-rs9658625, DNAH11-rs11768670, MVK-rs2287218 and CBS-rs234706, the presence of the minor allele was associated with a decrease in intestinal cholesterol absorption efficiency, as illustrated by a negative regression coefficient (Table 2).

SOAT2 encodes for sterol O-acyltransferase 2 but is also known under the alternative name ACAT2 (acyl-Coenzyme A: cholesterol acyltransferase 2; which is different from acetylCoenzyme A acetyltransferase 2, ACAT2). SOAT2 is a membrane-bound protein, mainly localized in enterocytes, that converts free cholesterol to cholesteryl esters for they packaging into chylomicrons [50] and whose deficiency in mice has been shown to lead to a decrease in cholesterol absorption efficiency and resistance to diet-induced hypercholesterolemia [51]. Yoshida et al. reported that the minor allele of SOAT2-rs9658625 was protective against chronic kidney disease in Japanese individuals with hypertension [52]. Since patients with chronic kidney disease have been shown to exhibit higher cholesterol absorption efficiency compared to healthy individuals [53], the negative association we find here between SOAT2-rs9658625 and cholesterol absorption is in agreement with results from Yoshida et al.. In addition, this SNP has been reported to be protective against dyslipidaemia in healthy Chinese [54].

For DNAH11 and GPAM, no precise biological mechanisms in relation to cholesterol metabolism are known but these genes were included as candidate genes following the association of some of their variants with circulating cholesterol concentration in a genomewide association study [55].

Hepatic lipase, encoded by the LIPC gene, is involved in the metabolism of HDL: by hydrolysing HDL triglycerides and phospholipids, it promotes the generation of smaller, denser HDL particles, which increase cholesterol efflux [56] and could thus also increase cholesterol reverse transport $[57,58]$. There is no previous report of an association of $L I P C$-rs690 with circulating TC or LDL-C concentrations. 
$M V K$ is involved in de novo cholesterol synthesis [59]. One case-control study showed that $M V K$-rs 2287218 is associated with an increased risk of coronary heart disease and ischemic stroke in a Chinese population, together with a slight decrease in HDL-cholesterol concentration in the controls [60]. Since there is an inverse relationship between cholesterol absorption and synthesis [41], we can hypothesize that the increase in de novo cholesterol synthesis suggested by this study could be related to a concomitant decrease in cholesterol absorption, as shown by the negative regression coefficient in our regression model.

$A P O E$ encodes for apolipoprotein $\mathrm{E}$, which plays a critical role in the intravascular transport and blood clearance of lipids. ApoE exists as three major isoforms, ApoE2, ApoE3 and ApoE4, which are the products of two SNPs, rs7412 and rs429358 [61]. Here, we show that APOE-rs7412 SNP, which determines the APOE2 isoform, is associated with an increase in the D7-cholesterol response. Results from other studies have yielded fairly conflicting results concerning the effect of APOE on cholesterol absorption $[39,49,62]$.

$C B S$ encodes for cystathionine beta-synthase, an enzyme that catalyses the conversion of homocysteine to cystathionine, the first step in the transsulfuration pathway. $C B S$-rs234706 was found to be associated with an increase in TC and LDL-C concentrations in participants from the Malmö Diet and Cancer Cardiovascular Cohort [63]. There is no known biological mechanism in relation to cholesterol metabolism although it has been linked to altered lipoprotein metabolism in mice [64] and expression levels of key enzymes involved in cholesterol synthesis in ovarian cancer cell lines [65].

$W R N$ was selected as a candidate gene because a SNP in this gene was reported to affect cholesterol efflux in vitro and to correlate with cholesterol concentrations in vivo [66].

Of note, we did not find any associated SNP in NPC1L1, which encodes for the main intestinal apical transporter of cholesterol and for which inactivating mutations have recently been associated with lower LDL-C concentrations and protection from CVD [21]. In fact, these mutations are rare, the most frequent having a minor allele frequency of only $0.02 \%$ (meaning one in every 5000 individuals is a heterozygous carrier), and were thus not present on the Beadchips (which usually have SNPs with minor allele frequency $>1 \%$ ).

We acknowledge some limitations of the study. It is likely that other SNPs that have a significant effect on cholesterol bioavailability were not entered in the statistical analysis because they are not present on the BeadChips, they were excluded from the analysis, or simply because they are located in other genes than the selected ones. Moreover, these findings need to be tested in other populations of greater size (based on e.g. sex, age, or genetic backgrounds). 
To conclude, our results show that, in this sample of healthy adult males, the relatively high interindividual variability in cholesterol bioavailability, is significantly associated with a combination of 8 exonic SNPs (SOAT2-rs9658625, DNAH11-rs11768670, LIPC-rs690, MVKrs2287218, GPAM-rs10787428, APOE-rs7412, CBS-rs234706 and $W R N$-rs1801196) located in genes involved in lipid metabolism and transport. Future studies based on the present data and model, as well as identification of other genes/SNPs involved in cholesterol bioavailability, will contribute to improve the quality of this association, with the ultimate objective to provide clinicians with an accurate and validated genetic tool to predict cholesterol bioavailability and thus provide them with additional information in order to personalize dietary/pharmacological treatment strategy to prevent CVD. 


\section{Author contributions}

$\mathrm{PB}, \mathrm{EW}$ and DL designed the research; $\mathrm{MN}$ and EW conducted the clinical research; EW and AN analysed D7-cholesterol by GC-MS; CDes, RV, MCA, PEM and PB analyzed data; CDes and CDef performed statistical analyses; CDes and PB wrote the paper and had primary responsibility for final content of the manuscript. All authors read and approved the final manuscript.

\section{Funding}

The present work has received research funding from the European Community's Sixth Framework Programme. The funding was attributed to the Lycocard project ( $\left.{ }^{\circ} 016213\right)$ which was an Integrated Project within the framework of the "Food Quality and Safety" program. This publication reflects only the view of the authors. The Lycocard community is not liable for any use that may be made of the results.

\section{Conflict of interest}

The authors have declared no conflicts of interest. 


\section{REFERENCES}

[1] Ference, B. A., Yoo, W., Alesh, I., Mahajan, N., et al., Effect of long-term exposure to lower low-density lipoprotein cholesterol beginning early in life on the risk of coronary heart disease: a Mendelian randomization analysis. J Am Coll Cardiol 2012, 60, 2631-2639.

[2] Stamler, J., Daviglus, M. L., Garside, D. B., Dyer, A. R., et al., Relationship of baseline serum cholesterol levels in 3 large cohorts of younger men to long-term coronary, cardiovascular, and all-cause mortality and to longevity. JAMA 2000, 284, 311-318.

[3] Miettinen, T. A., Gylling, H., Strandberg, T., Sarna, S., Baseline serum cholestanol as predictor of recurrent coronary events in subgroup of Scandinavian simvastatin survival study. Finnish 4S Investigators. BMJ 1998, 316, 1127-1130.

[4] Hoenig, M. R., Rolfe, B. E., Campbell, J. H., Cholestanol: a serum marker to guide LDL cholesterol-lowering therapy. Atherosclerosis 2006, 184, 247-254.

[5] Gylling, H., Plat, J., Turley, S., Ginsberg, H. N., et al., Plant sterols and plant stanols in the management of dyslipidaemia and prevention of cardiovascular disease. Atherosclerosis 2014, $232,346-360$.

[6] Jenkins, D. J., Jones, P. J., Lamarche, B., Kendall, C. W., et al., Effect of a dietary portfolio of cholesterol-lowering foods given at 2 levels of intensity of dietary advice on serum lipids in hyperlipidemia: a randomized controlled trial. Jama 2011, 306, 831-839.

[7] Grundy, S. M., Does Dietary Cholesterol Matter? Curr Atheroscler Rep 2016, 18, 68.

[8] Lutjohann, D., Stellaard, F., Mulder, M. T., Sijbrands, E. J. G., Weingartner, O., The emerging concept of "individualized cholesterol-lowering therapy": A change in paradigm. Pharmacol Ther 2019, 199, 111-116.

[9] Sudhop, T., Lutjohann, D., Kodal, A., Igel, M., et al., Inhibition of intestinal cholesterol absorption by ezetimibe in humans. Circulation 2002, 106, 1943-1948. 
[10] Insull, W., Jr., Clinical utility of bile acid sequestrants in the treatment of dyslipidemia: a scientific review. South Med J 2006, 99, 257-273.

[11] Garcia-Calvo, M., Lisnock, J., Bull, H. G., Hawes, B. E., et al., The target of ezetimibe is Niemann-Pick C1-Like 1 (NPC1L1). Proc Natl Acad Sci U S A 2005, 102, 8132-8137.

[12] Cannon, C. P., Blazing, M. A., Giugliano, R. P., McCagg, A., et al., Ezetimibe Added to Statin Therapy after Acute Coronary Syndromes. N Engl J Med 2015, 372, 2387-2397.

[13] Ouchi, Y., Sasaki, J., Arai, H., Yokote, K., et al., Ezetimibe Lipid-Lowering Trial on Prevention of Atherosclerotic Cardiovascular Disease in 75 or Older (EWTOPIA 75): A Randomized, Controlled Trial. Circulation 2019, 140, 992-1003.

[14] Mozaffarian, D., Dietary and Policy Priorities for Cardiovascular Disease, Diabetes, and Obesity: A Comprehensive Review. Circulation 2016, 133, 187-225.

[15] Bosner, M. S., Lange, L. G., Stenson, W. F., Ostlund, R. E., Jr., Percent cholesterol absorption in normal women and men quantified with dual stable isotopic tracers and negative ion mass spectrometry. J Lipid Res 1999, 40, 302-308.

[16] Alphonse, P. A., Jones, P. J., Revisiting Human Cholesterol Synthesis and Absorption: The Reciprocity Paradigm and its Key Regulators. Lipids 2016, 51, 519-536.

[17] Warrier, M., Shih, D. M., Burrows, A. C., Ferguson, D., et al., The TMAO-Generating Enzyme Flavin Monooxygenase 3 Is a Central Regulator of Cholesterol Balance. Cell Rep 2015.

[18] Wang, D. Q., Regulation of intestinal cholesterol absorption. Annu Rev Physiol 2007, 69, 221-248.

[19] Gylling, H., Miettinen, T. A., Inheritance of cholesterol metabolism of probands with high or low cholesterol absorption. J Lipid Res 2002, 43, 1472-1476.

[20] Silbernagel, G., Chapman, M. J., Genser, B., Kleber, M. E., et al., High intestinal cholesterol absorption is associated with cardiovascular disease and risk alleles in ABCG8 and 
ABO: evidence from the LURIC and YFS cohorts and from a meta-analysis. $J$ Am Coll Cardiol 2013, 62, 291-299.

[21] Myocardial Infarction Genetics Consortium, I., Stitziel, N. O., Won, H. H., Morrison, A. C., et al., Inactivating mutations in NPC1L1 and protection from coronary heart disease. $N$ Engl J Med 2014, 371, 2072-2082.

[22] Beaumier-Gallon, G., Dubois, C., Senft, M., Vergnes, M. F., et al., Dietary cholesterol is secreted in intestinally derived chylomicrons during several subsequent postprandial phases in healthy humans. Am J Clin Nutr 2001, 73, 870-877.

[23] Wang, Y., Vanstone, C. A., Parsons, W. D., Jones, P. J., Validation of a single-isotopelabeled cholesterol tracer approach for measuring human cholesterol absorption. Lipids 2004, $39,87-91$.

[24] Wolff, E., Vergnes, M. F., Kaloustian, J., Abou, L., et al., A new approach to overcome natural cholesterol interference during simultaneous determination of two stable isotopeenriched cholesterol tracers in human plasma. Rapid Commun Mass Spectrom 2007, 21, 31753179 .

[25] Ostlund, R. E., Jr., Spilburg, C. A., Stenson, W. F., Sitostanol administered in lecithin micelles potently reduces cholesterol absorption in humans. Am J Clin Nutr 1999, 70, 826-831. [26] Borel, P., Desmarchelier, C., Nowicki, M., Bott, R., A Combination of Single-Nucleotide Polymorphisms Is Associated with Interindividual Variability in Dietary beta-Carotene Bioavailability in Healthy Men. J Nutr 2015, 145, 1740-1747.

[27] Cordell, H. J., Clayton, D. G., Genetic association studies. Lancet 2005, 366, 1121-1131.

[28] Lettre, G., Lange, C., Hirschhorn, J. N., Genetic model testing and statistical power in population-based association studies of quantitative traits. Genet Epidemiol 2007, 31, 358-362.

[29] Lewis, C. M., Genetic association studies: design, analysis and interpretation. Brief Bioinform 2002, 3, 146-153. 
[30] Purcell, S., Neale, B., Todd-Brown, K., Thomas, L., et al., PLINK: a tool set for wholegenome association and population-based linkage analyses. Am J Hum Genet 2007, 81, 559575.

[31] Brindle, J. T., Antti, H., Holmes, E., Tranter, G., et al., Rapid and noninvasive diagnosis of the presence and severity of coronary heart disease using 1H-NMR-based metabonomics. Nat Med 2002, 8, 1439-1444.

[32] Borel, P., Desmarchelier, C., Nowicki, M., Bott, R., Tourniaire, F., Can genetic variability in alpha-tocopherol bioavailability explain the heterogeneous response to alpha-tocopherol supplements? Antioxid Redox Signal 2015, 22, 669-678.

[33] Desmarchelier, C., Martin, J. C., Planells, R., Gastaldi, M., et al., The postprandial chylomicron triacylglycerol response to dietary fat in healthy male adults is significantly explained by a combination of single nucleotide polymorphisms in genes involved in triacylglycerol metabolism. J Clin Endocrinol Metab 2014, 99, E484-488.

[34] Beaumier-Gallon, G., Lanfranchi, J., Vergnes, M.-F., Lairon, D., et al., Method for simultaneous measurements of traces of heptadeuterated cholesterol and cholesterol by gas chromatography-mass spectrometry: application in humans. Journal of Chromatography B: Biomedical Sciences and Applications 1998, 718, 23-32.

[35] MacKay, D., Jones, P. J. H., Evaluation of methods for the determination of cholesterol absorption and synthesis in humans. Atherosclerosis 2011, 218, 253-262.

[36] Ostlund, R. E., Jr., Racette, S. B., Stenson, W. F., Inhibition of cholesterol absorption by phytosterol-replete wheat germ compared with phytosterol-depleted wheat germ. Am J Clin Nutr 2003, 77, 1385-1389.

[37] Ference, B. A., Ginsberg, H. N., Graham, I., Ray, K. K., et al., Low-density lipoproteins cause atherosclerotic cardiovascular disease. 1. Evidence from genetic, epidemiologic, and 
clinical studies. A consensus statement from the European Atherosclerosis Society Consensus Panel. Eur Heart J 2017, 38, 2459-2472.

[38] Kesaniemi, Y. A., Miettinen, T. A., Cholesterol absorption efficiency regulates plasma cholesterol level in the Finnish population. Eur J Clin Invest 1987, 17, 391-395.

[39] Silbernagel, G., Fauler, G., Hoffmann, M. M., Lutjohann, D., et al., The associations of cholesterol metabolism and plasma plant sterols with all-cause and cardiovascular mortality. $J$ Lipid Res 2010, 51, 2384-2393.

[40] Matthan, N. R., Pencina, M., LaRocque, J. M., Jacques, P. F., et al., Alterations in cholesterol absorption/synthesis markers characterize Framingham offspring study participants with CHD. J Lipid Res 2009, 50, 1927-1935.

[41] Grundy, S. M., Ahrens, E. H., Jr., Davignon, J., The interaction of cholesterol absorption and cholesterol synthesis in man. J Lipid Res 1969, 10, 304-315.

[42] Berger, S., Raman, G., Vishwanathan, R., Jacques, P. F., Johnson, E. J., Dietary cholesterol and cardiovascular disease: a systematic review and meta-analysis. Am J Clin Nutr 2015, 102, 276-294.

[43] Lecerf, J. M., de Lorgeril, M., Dietary cholesterol: from physiology to cardiovascular risk. Br J Nutr 2011, 106, 6-14.

[44] Dehghan, M., Mente, A., Rangarajan, S., Mohan, V., et al., Association of egg intake with blood lipids, cardiovascular disease, and mortality in 177,000 people in 50 countries. Am J Clin Nutr 2020, 111, 795-803.

[45] Zhong, V. W., Van Horn, L., Cornelis, M. C., Wilkins, J. T., et al., Associations of Dietary Cholesterol or Egg Consumption With Incident Cardiovascular Disease and Mortality. Jama 2019, 321, 1081-1095. 
[46] Calandra, S., Tarugi, P., Speedy, H. E., Dean, A. F., et al., Mechanisms and genetic determinants regulating sterol absorption, circulating LDL levels, and sterol elimination: implications for classification and disease risk. J Lipid Res 2011, 52, 1885-1926.

[47] Gylling, H., Hallikainen, M., Pihlajamaki, J., Agren, J., et al., Polymorphisms in the ABCG5 and ABCG8 genes associate with cholesterol absorption and insulin sensitivity. J Lipid Res 2004, 45, 1660-1665.

[48] Maeda, T., Honda, A., Ishikawa, T., Kinoshita, M., et al., A SNP of NPC1L1 affects cholesterol absorption in Japanese. J Atheroscler Thromb 2010, 17, 356-360.

[49] Kesaniemi, Y. A., Ehnholm, C., Miettinen, T. A., Intestinal cholesterol absorption efficiency in man is related to apoprotein E phenotype. J Clin Invest 1987, 80, 578-581.

[50] Chang, T. Y., Li, B. L., Chang, C. C., Urano, Y., Acyl-coenzyme A:cholesterol acyltransferases. Am J Physiol Endocrinol Metab 2009, 297, E1-9.

[51] Buhman, K. K., Accad, M., Novak, S., Choi, R. S., et al., Resistance to diet-induced hypercholesterolemia and gallstone formation in ACAT2-deficient mice. Nat Med 2000, 6, $1341-1347$.

[52] Yoshida, T., Kato, K., Yokoi, K., Watanabe, S., et al., Association of candidate gene polymorphisms with chronic kidney disease in Japanese individuals with hypertension. Hypertens Res 2009, 32, 411-418.

[53] Rogacev, K. S., Pinsdorf, T., Weingartner, O., Gerhart, M. K., et al., Cholesterol synthesis, cholesterol absorption, and mortality in hemodialysis patients. Clin J Am Soc Nephrol 2012, 7, 943-948.

[54] He, X., Lu, Y., Saha, N., Yang, H., Heng, C. K., Acyl-CoA: cholesterol acyltransferase-2 gene polymorphisms and their association with plasma lipids and coronary artery disease risks. Hum Genet 2005, 118, 393-403. 
[55] Teslovich, T. M., Musunuru, K., Smith, A. V., Edmondson, A. C., et al., Biological, clinical and population relevance of 95 loci for blood lipids. Nature 2010, 466, 707-713.

[56] Jansen, H., Verhoeven, A. J., Sijbrands, E. J., Hepatic lipase: a pro- or anti-atherogenic protein? J Lipid Res 2002, 43, 1352-1362.

[57] Annema, W., Tietge, U. J., Role of hepatic lipase and endothelial lipase in high-density lipoprotein-mediated reverse cholesterol transport. Curr Atheroscler Rep 2011, 13, 257-265.

[58] Escola-Gil, J. C., Chen, X., Julve, J., Quesada, H., et al., Hepatic lipase- and endothelial lipase-deficiency in mice promotes macrophage-to-feces RCT and HDL antioxidant properties. Biochim Biophys Acta 2013, 1831, 691-697.

[59] Horton, J. D., Shah, N. A., Warrington, J. A., Anderson, N. N., et al., Combined analysis of oligonucleotide microarray data from transgenic and knockout mice identifies direct SREBP target genes. Proc Natl Acad Sci U S A 2003, 100, 12027-12032.

[60] Wang, D. S., Yin, R. X., Li, K. G., Lu, L., et al., Association between the MVK rs2287218 SNP and the risk of coronary heart disease and ischemic stroke: A case-control study. Biosci Trends 2018, 12, 403-411.

[61] Phillips, M. C., Apolipoprotein E isoforms and lipoprotein metabolism. IUBMB Life 2014, 66, 616-623.

[62] Von Bergmann, K., Lutjohann, D., Lindenthal, B., Steinmetz, A., Efficiency of intestinal cholesterol absorption in humans is not related to apoE phenotype. J Lipid Res 2003, 44, 193 197.

[63] Sharma, A., Gulbahce, N., Pevzner, S. J., Menche, J., et al., Network-based analysis of genome wide association data provides novel candidate genes for lipid and lipoprotein traits. Mol Cell Proteomics 2013, 12, 3398-3408. 
[64] Liao, D., Tan, H., Hui, R., Li, Z., et al., Hyperhomocysteinemia decreases circulating highdensity lipoprotein by inhibiting apolipoprotein A-I Protein synthesis and enhancing HDL cholesterol clearance. Circ Res 2006, 99, 598-606.

[65] Chakraborty, P. K., Xiong, X., Mustafi, S. B., Saha, S., et al., Role of cystathionine beta synthase in lipid metabolism in ovarian cancer. Oncotarget 2015, 6, 37367-37384.

[66] Berube, J., Garand, C., Lettre, G., Lebel, M., The non-synonymous polymorphism at position 114 of the WRN protein affects cholesterol efflux in vitro and correlates with cholesterol levels in vivo. Exp Gerontol 2013, 48, 533-538. 


\section{FIGURE LEGENDS}

Figure 1. Plasma D7-cholesterol response $40 \mathrm{~h}$ after the ingestion of a D7-cholesterol-rich test snack.

Participants were sorted by increasing plasma D7-cholesterol response, i.e. the ratio plasma D7cholesterol concentration/fasting plasma TC concentration.

Figure 2. D7-cholesterol response according to the genotype at the $8 \mathrm{SNPs}$ in the selected Partial Least Squares regression model.

Corresponding p-values can be found in Table 2. 


\section{TABLES}

Table 1. Participant characteristics. ${ }^{\mathrm{a}}$

\begin{tabular}{|c|c|}
\hline Characteristic & Mean \\
\hline Age (y) & $33.1 \pm 2.1$ \\
\hline Weight (kg) & $73.5 \pm 1.3$ \\
\hline $\mathrm{BMI}\left(\mathrm{kg} / \mathrm{m}^{2}\right)$ & $22.9 \pm 0.3$ \\
\hline Total cholesterol $(\mathrm{mmol} / \mathrm{L})^{\mathrm{b}}$ & $4.3 \pm 0.1$ \\
\hline HDL-cholesterol $(\mathrm{mmol} / \mathrm{L})^{\mathrm{b}}$ & $1.2 \pm 0.0$ \\
\hline LDL-cholesterol $(\mathrm{mmol} / \mathrm{L})^{\mathrm{b}}$ & $2.8 \pm 0.1$ \\
\hline Triglycerides $(\mathrm{mmol} / \mathrm{L})^{\mathrm{b}}$ & $0.8 \pm 0.1$ \\
\hline Glucose $(\mathrm{mmol} / \mathrm{L})^{\mathrm{b}}$ & $4.8 \pm 0.1$ \\
\hline Haemoglobin $(\mathrm{g} / \mathrm{dL})^{\mathrm{b}}$ & $15.1 \pm 0.1$ \\
\hline
\end{tabular}

${ }^{a} \mathrm{n}=39$.

${ }^{\mathrm{b}}$ Fasting plasma variables. 
Table 2. Genes and SNPs significantly associated with the plasma D7-cholesterol response following univariate analysis., ${ }^{a, b}$

\begin{tabular}{|c|c|c|c|c|c|c|}
\hline Gene $^{c}$ & SNP rs & Alleles & $\begin{array}{l}\text { Minor allele } \\
\text { Frequency }^{d}\end{array}$ & $\begin{array}{l}\text { Coding } \\
\text { status }\end{array}$ & $\begin{array}{l}\text { Unstandardized } \\
\text { regression } \\
\text { coefficient }^{\mathrm{e}}\end{array}$ & p-value ${ }^{e}$ \\
\hline \multicolumn{7}{|c|}{ Additive model ${ }^{\mathrm{f}}$} \\
\hline$L I P C$ & rs690 & $\mathrm{G}>\mathrm{T}$ & $\mathrm{G}=0.427$ & & $0.213 \pm 0.083$ & 0.015 \\
\hline GPAM & rs10787428 & $\mathrm{T}>\mathrm{C}$ & $\mathrm{T}=0.417$ & NonSyn ${ }^{g}$ & $0.229 \pm 0.100$ & 0.028 \\
\hline$W R N$ & rs1801195 & $\mathrm{G}>\mathrm{T}$ & $\mathrm{T}=0.449$ & NonSyn & $0.205 \pm 0.097$ & 0.042 \\
\hline$W R N$ & rs1800392 & $\mathrm{G}>\mathrm{T}$ & $\mathrm{T}=0.452$ & & $0.194 \pm 0.095$ & 0.049 \\
\hline \multicolumn{7}{|c|}{ Dominant model ${ }^{\mathrm{h}}$} \\
\hline SOAT2 & rs9658625 & $A>G$ & $\mathrm{G}=0.073$ & NonSyn & $-0.468 \pm 0.165$ & 0.007 \\
\hline DNAH11 & rs11768670 & $\mathrm{G}>\mathrm{A}$ & $A=0.223$ & NonSyn & $-0.320 \pm 0.121$ & 0.012 \\
\hline$M V K$ & rs2287218 & $\mathrm{C}>\mathrm{T}$ & $\mathrm{T}=0.176$ & & $-0.299 \pm 0.128$ & 0.025 \\
\hline$L I P C$ & rs690 & $\mathrm{G}>\mathrm{T}$ & $\mathrm{G}=0.427$ & & $0.307 \pm 0.133$ & 0.027 \\
\hline$A P O E$ & rs7412 & $\mathrm{C}>\mathrm{T}$ & $\mathrm{T}=0.087$ & NonSyn & $0.340 \pm 0.153$ & 0.032 \\
\hline$C B S$ & rs234706 & $\mathrm{G}>\mathrm{A}$ & $A=0.328$ & & $-0.278 \pm 0.126$ & 0.033 \\
\hline$W R N$ & rs1801196 & $\mathrm{C}>\mathrm{T}$ & $\mathrm{T}=0.291$ & & $0.271 \pm 0.124$ & 0.036 \\
\hline DNAH11 & rs6965750 & $\mathrm{G}>\mathrm{A}$ & $A=0.140$ & NonSyn & $-0.274 \pm 0.130$ & 0.041 \\
\hline DNAH11 & rs9639393 & $A>G$ & $A=0.396$ & & $-0.255 \pm 0.125$ & 0.048 \\
\hline
\end{tabular}

a Plasma D7-cholesterol response represents the ratio plasma D7-cholesterol concentration/fasting plasma TC concentration.

${ }^{\mathrm{b}}$ SNPs are ranked by decreasing $\mathrm{p}$-values.

${ }^{c}$ See Supporting Information Table S1 for gene names.

${ }^{\mathrm{d}}$ Minor allele frequency were retrieved from dbSNP (https://www.ncbi.nlm.nih.gov/snp/) using the Allele Frequency Aggregator (ALFA) dataset (pooled allele frequency data from $\mathrm{dbSNP}$ and the $\mathrm{dbGaP}$ ) in the global population.

${ }^{\mathrm{d}}$ Unstandardized regression coefficients (B coefficients) represent the mean change in the D7cholesterol response for each additional copy of the minor allele under the additive model and in the presence of the minor allele under the dominant model.

${ }^{\mathrm{e}}$ Wald test asymptotic p-value. 
${ }^{\mathrm{f}}$ The additive model assumes that there is a uniform, linear increase or decrease in the quantitative trait for each copy of the minor allele.

${ }^{\mathrm{g}}$ NonSyn: non synonymous variation. All SNPs were otherwise coding variants (i.e. located in exons).

${ }^{\mathrm{h}}$ For SNPs under the dominant model, participants homozygous for the lesser frequent allele were grouped with heterozygous participants and were compared with participants homozygous for the more frequent allele. 
Table 3. Combination of SNPs associated with the plasma D7-cholesterol response. ${ }^{\text {a }}$

\begin{tabular}{llll}
\hline Gene $^{\mathbf{b}}$ & SNP & VIP value & $\begin{array}{l}\text { Regression } \\
\text { coefficient }^{\mathbf{c}}\end{array}$ \\
\hline SOAT2 & rs9658625 & 1.143 & -0.292 \\
DNAH11 & rs11768670 & 1.084 & -0.200 \\
LIPC & rs690 & 1.051 & 0.133 \\
MVK & rs2287218 & 0.971 & -0.187 \\
GPAM & rs10787428 & 0.955 & 0.143 \\
APOE & rs7412 & 0.931 & 0.213 \\
CBS & rs234706 & 0.927 & -0.174 \\
WRN & rs1801196 & 0.913 & 0.169 \\
\hline
\end{tabular}

${ }^{a}$ Variables were ranked by decreasing variable importance in the projection (VIP) value, which estimates the contribution of each SNP in the projection used in the PLS regression model, and thus in the studied phenotype.

${ }^{\mathrm{b}}$ See Supporting Information Table $\mathbf{S 1}$ for gene names.

${ }^{c}$ Regression coefficient are for untransformed variables and represent the mean change in the D7-cholesterol response for each additional copy of the minor allele under the additive model and in the presence of the minor allele under the dominant model.

${ }^{\mathrm{d}}$ These SNPs were entered under the additive model. 